\title{
Application of Kaiser Model-Based Disaster Vulnerability Analysis in Pediatric Clinics of Tertiary General Hospitals
}

\author{
Qian NIU, Yu-lian ZHANG*, Fu-yong JIAO*, Rui LI, Zhi-long MU, Jing-hua Gao and Xi-pin Zhang \\ Children' Hospital of Shaanxi Provincial People's Hospital, China
}

*Corresponding author: Yu-lian ZHANG, Deputy Director of Shaanxi Provincial

People's Hospital, Xi'an, China.

Fu-yong JIAO, Children's Hospital of Shaanxi Provincial People's Hospital, Xi'an,

China, Email: jiaofy@yeah.net

Received Date: January 25, 2021

Published Date: February 23, 2021

Abstract

Objective: To find out the risk events of pediatric outpatients by carrying out disaster vulnerability analysis, improve the emergency management and disposal capabilities of pediatric outpatients, and effectively avoid and deal with disaster risk events.

Methods: The Delphi method was used to screen for risk events, and the Kaiser model was used to design a personalized questionnaire to analyze the vulnerability of pediatric outpatient services.

Results: Through analysis, sorted out the top ten types of risk events, drug safety hazards (42.57\%), patient identification error events (40.43\%), patient falls / falls (39.93\%), and violent medical disputes (38.84\%), fire (38.37\%), emergency life support system failure (38.08\%), information system failure (35.14\%), infectious disease epidemic (33.72\%), medical gas failure (32.33\%), air-conditioning failure (29.36\%).

Conclusion: Disaster vulnerability analysis can more realistically reflect the key and difficult issues of emergency management of pediatric outpatients, provide objective evidence for pediatric outpatient emergency management planning, and improve the risk management of departments.

Keywords: Kaiser model; Vulnerability analysis; Pediatric clinic; Emergency management; Risk management

\section{Introduction}

Shaanxi Provincial People's Hospital is a tertiary A general hospital integrating medicine, teaching, and research. The pediatric outpatient department is a large-scale comprehensive outpatient clinic integrating outpatient, emergency, laboratory tests, examinations, outpatient infusion, and atomization. The staff composition of the workplace is complicated, the medical treatment tasks are heavy, the visits and treatment links are many, and it is time-consuming and easy to intensify the medical treatment. Potential contradictions between patients, trigger medical-related emergency disasters.

The vulnerability of the hospital refers to the possibility of the hospital being affected by various potential disasters and the ability to withstand disasters [1]. Hazard vulnerability analysis (HVA) is an important tool for assessing and preventing medical disasters. It determines the nature and impact of disasters, analyzes and examines the ability to resist risks, and takes corresponding measures for weak links to reduce and reduce losses. An analysis method [2]. Kaiser model is a tool for vulnerability analysis of medical institutions developed by Kaiser Permanente Medical Group in the United States [3]. It has the advantages of professional classification, complete content and wide application range. It is currently the most widely used statistical method. It calculates various risk events. The probability and the hazard of the incident are analyzed and processed with the probability of occurrence as the weight, combined with the evaluation of the hazard level, and the risk coefficient of related events is [4-6]. Therefore, this study uses the Kaiser model to analyze the vulnerability of pediatric 
outpatients and conduct targeted risk management. The report is as follows.

\section{Materials and Methods}

Materials: This study invited the outpatient office, the nursing department, the director of the logistics security department, the director of pediatrics, and the head nurse to form an expert group, and 52 risk events in 4 dimensions mentioned in the "Chinese Hospital Review Practice" were used as the preliminary screening indicators [7], combined with the geographical particularity of the pediatric clinic, the hospital situation, the scope of diagnosis and treatment services, etc., through brainstorming and Delphi method screening, it is finally determined as 22 risk events in 4 dimensions, which will not meet the actual risks of the undergraduate office in the region Events, such as earthquakes, hurricanes, volcanic eruptions, etc., are eliminated and sorted out according to actual clinical items to determine risk events (Table 1).

Table 1: Sources and classifications of disaster vulnerability in the pediatric outpatient clinic of a tertiary hospital.

\begin{tabular}{|c|c|}
\hline Event Classification & Risk Event \\
\hline Natural disasters & Storms, fires \\
\hline Technical accidents & $\begin{array}{l}\text { Power failures, information system failures, water supply system failures, air conditioning failures, medical gas failures, } \\
\text { drug safety hazards, communication failures, emergency life support system failures }\end{array}$ \\
\hline Man-made injuries & $\begin{array}{l}\text { Mass casualties, violent medical disputes, food safety incidents, infectious disease outbreaks, hospital infection outbreaks, } \\
\text { patients falling/falling from bed, suffocation, mistaking, incorrect identification, and major theft }\end{array}$ \\
\hline $\begin{array}{l}\text { Dangerous goods injury } \\
\text { category Hazardous }\end{array}$ & chemical injury, flammable and explosive product injury \\
\hline
\end{tabular}

Design the questionnaire: Based on the disaster vulnerability of the pediatric outpatient clinic and its source, and use the Kaiser model risk assessment matrix to design the pediatric outpatient disaster vulnerability analysis questionnaire. The content includes the probability of disaster events, personnel injuries, property losses, service impact, emergency preparedness, Seven aspects including internal response and external response. (1) Possibility of occurrence, that is, the probability of occurrence: refer to the known data in recent years, past historical data, etc.; (2) Personnel damage: mainly consider the possible staff casualties, the casualties of children and parents, and the injured (3) Asset loss: mainly calculate the cost of updating after the incident, the cost of establishing temporary replacement facilities, the cost of maintenance, the time required for normalization, etc.; (4) The impact of operational services : Mainly concerned about the interruption of normal work, the interruption of the supply of key materials, the interruption of external services, the attrition of employees, the obstruction of the arrival of patients, the failure to perform the contract, the failure to comply with the regulations, possible legal disputes, loss of public reputation and image As well as the increase in the financial burden of the hospital, etc.; (5) Emergency preparedness: Mainly focus on whether the emergency

Table 2: Scoring criteria for disaster vulnerability analysis. plan is perfect, whether emergency drills are frequently carried out, whether necessary training is provided to the staff, the situation of emergency supplies and emergency support, etc.; (6) Internal response: the internal response capability, which mainly considers the time required to make an effective response, whether the current types and quantities of materials can meet the needs, the staff's mastery of relevant skills, the estimated severity and duration of the incident, and whether there is backup (7) External response: including national and local emergency response capabilities, mutual assistance agreements signed by relevant agencies, coordination with hospitals, community volunteers, and emergency response signed with material supply agencies Supply plan or contract status, etc.

Drafting the scoring standards: According to the designed questionnaire items, combined with the policies of the hospital and related rules and regulations, each indicator is divided into 4 levels, with $0,1,2$, and 3 assigned respectively, and the probability of occurrence is 0 - Level 3, level 3 is the highest; personnel injury, property damage, and service impact in serious items are divided into 0-3 levels, and level 3 is the highest, while emergency preparedness, internal response, and external support are divided into 0-3 levels, and level 3 is the lowest (Table 2).

\begin{tabular}{|c|c|c|c|c|c|c|c|}
\hline \multirow{2}{*}{$\begin{array}{l}\text { Danger- } \\
\text { ous Event }\end{array}$} & \multirow[b]{2}{*}{ Likelihood } & \multicolumn{6}{|c|}{ Severity } \\
\hline & & $\begin{array}{l}\text { Personnel } \\
\text { Impact }\end{array}$ & $\begin{array}{l}\text { Property } \\
\text { Impact }\end{array}$ & Operational Impact & $\begin{array}{c}\text { Emergency Pre- } \\
\text { paredness }\end{array}$ & $\begin{array}{l}\text { Internal Re- } \\
\text { sponse }\end{array}$ & $\begin{array}{l}\text { External Re- } \\
\text { sponse }\end{array}$ \\
\hline & $\begin{array}{c}\text { Probability of } \\
\text { occurrence }\end{array}$ & $\begin{array}{l}\text { Medical staff } \\
\text { injury }\end{array}$ & $\begin{array}{l}\text { Hospital prop- } \\
\text { erty damage }\end{array}$ & $\begin{array}{l}\text { Hospital service } \\
\text { impact }\end{array}$ & $\begin{array}{l}\text { Prepare situation } \\
\text { in advance }\end{array}$ & $\begin{array}{l}\text { Time, efficiency, } \\
\text { resources }\end{array}$ & $\begin{array}{l}\text { Exchange, mutual } \\
\text { assistance, sup- } \\
\text { port }\end{array}$ \\
\hline \multirow{4}{*}{$\begin{array}{l}\text { Core Stan- } \\
\quad \text { dard }\end{array}$} & $0=\mathrm{N} / \mathrm{A}$ & $0=\mathrm{N} / \mathrm{A}$ & $0=\mathrm{N} / \mathrm{A}$ & $0=\mathrm{N} / \mathrm{A}$ & $0=\mathrm{N} / \mathrm{A}$ & $0=\mathrm{N} / \mathrm{A}$ & $0=\mathrm{N} / \mathrm{A}$ \\
\hline & $1=$ low & $1=$ low & $1=$ low & $1=$ low & $1=$ high & $1=$ high & 1=high \\
\hline & $2=$ medium & $2=$ medium & $2=$ medium & $2=$ medium & $2=$ medium & $2=$ medium & $2=$ medium \\
\hline & $3=$ high & $3=$ high & $3=$ high & $3=$ high & $3=$ low $/$ none & $3=$ low $/$ none & $3=$ low $/$ none \\
\hline
\end{tabular}


Investigation and Statistics: According to the content and form of disaster vulnerability analysis and assessment and the filling requirements of the questionnaire, explain to all levels of personnel in the pediatric outpatient department, including administrative staff, logistics security, clinical, medical technology, etc., after understanding The questionnaire is issued, completed on the spot and taken back. 50 questionnaires were issued and 50 were recovered, with a recovery rate of $100 \%$; 48 were valid, with an effective rate of $96 \%$. The survey data is entered into Excel2007 for statistical analysis, and the calculation formula of relative risk based on Kaiser model combining probability and severity: relative risk $\%=($ probability $/ 3) \times\{$ (personal injury + property loss + service impact + emergency preparedness + internal Reaction + external

Table 3: Results of disaster vulnerability analysis reaction $) / 3 \times(3+3+3+3+3+3)\}$, calculate the relative risk value of each dangerous event, and sort it according to the relative risk value. The first order is to The main high-risk events of concern.

\section{Result}

According to the ranking of the relative risk value, the top ten disaster events faced by pediatric clinics are: drug safety hazards, patient identification errors, patient falling/falling, violent medical disputes, fires, emergency life support system failures, and information systems Malfunctions, epidemics of infectious diseases, medical gas malfunctions, air-conditioning malfunctions. The results show that it is mainly technical hazards and man-made hazards. Natural fire disasters cannot be ignored, and special attention must be paid (Table 3).

\begin{tabular}{|c|c|c|c|}
\hline Event Category & Risk Event Description & Relative Risk Value & Sort \\
\hline Natural disasters & fire & $38.37 \%$ & 5 \\
\hline \multirow{5}{*}{ Technical hazards } & Information system failure & $35.14 \%$ & 7 \\
\hline & Medical gas failure & $32.33 \%$ & 9 \\
\hline & Air conditioner malfunction & $29.36 \%$ & 10 \\
\hline & Drug safety hazards & $42.57 \%$ & 1 \\
\hline & Emergency life support system failure & $38.08 \%$ & 6 \\
\hline \multirow{4}{*}{ Man-made hazards } & Violent medical dispute & $38.84 \%$ & 4 \\
\hline & Patient falls/falls out of bed & $39.93 \%$ & 3 \\
\hline & Infectious disease & $33.72 \%$ & 8 \\
\hline & Patient identification error & $40.43 \%$ & 2 \\
\hline
\end{tabular}

According to this disaster vulnerability analysis, the above ten dangerous events in pediatrics are listed as key management items, and the Emergency Plan for Medication Errors, Emergency Plan for Failure of Hospital Information System, Emergency Plan for Infectious Disease Epidemics, and Hospital Central Three preplans of the Air Conditioning System Failure Emergency Plan, and the original plans such as the "Patient Fall/Bed Falling Emergency Plan", "Allergic Shock Rescue Emergency Plan", "Fire Emergency Plan", "Emergency Handling Plan for Power Failure" and other plans were revised Improve, formulate preventive measures, organize special training and emergency drills for medical staff in pediatric outpatient clinics to ensure that the medical staff in pediatric outpatient clinics pass the pass.

\section{Discussion}

The ranking of risk events reflects the characteristics of pediatric outpatient clinics, and has a certain degree of scientificity: Through the results of disaster vulnerability analysis, the main emergencies that may have an impact and the weak links of the current emergency management are further clarified, and these guides continuous improvement of emergency plans. , Improve the risk management of pediatric outpatient clinics. Table 3 shows that among the risk events in pediatric outpatient clinics, drug safety hazards are the most dangerous. This is in contrast to the drastic increase in the number of outpatients in recent years. There are many oral medications and infusion treatments for children, even for certain examinations. It is related to the occurrence of multiple adverse events when it comes to drugs. Therefore, it is necessary to formulate detailed risk prevention measures in terms of patient safe medication, strengthen the technical training and sense of responsibility of medical staff, and strengthen the quality control of links. Secondly, there are accidents of children's identity confirmation errors. There are many patients and parents are anxious. It is very easy to have children's identity confirmation errors during treatment, examination, medicine delivery, and injection. Medical staff are required to strictly implement the core system and conduct regular training and assessment. The third is the incident of children falling and falling from bed. There are many children, the outpatient space is small, and the children are very active. In recent years, there have been many children falling and falling from bed incidents. This should arouse great attention. These disasters have not only caused the children. Great harm, but also has a greater negative impact on the hospital. The pediatric outpatient and emergency department of our hospital is an independent two-story building that was 
transformed from an old building 5 years ago. Analysis shows that the relative risk of fire is as high as $38.38 \%$. It is the fourth most dangerous event in the pediatric outpatient department, prompting us to do a good job in fire emergency response. Plan and conduct regular drills.

In recent years, with the development of informatization, hospitals have generally implemented informatization electronic systems. Hospitals have become more and more dependent on informatization and the network. Informatization system obstacles and power system obstacles will bring greater damage. Important events that cannot be ignored by the hospital are consistent with the results of this analysis. Therefore, it is necessary to obtain strong support from the hospital to make emergency plans and training.

\section{Kaiser model can be used for disaster vulnerability analysis of key departments in hospitals: Disaster} vulnerability analysis is based on the actual situation of the hospital, investigating and researching disaster risk events that have occurred or may occur, by looking for problems that are vulnerable to danger, analyzing response measures, and formulating emergency plans to reduce risks and losses [8-10]. The Kaiser model evaluation method is concise, the evaluation result is objective, and it has good applicability $[3,11]$. For the first time, this study used the Kaiser model to analyze the disaster vulnerability of key disaster-prone pediatric clinics, and the results have been recognized and valued by everyone. The results of this study confirm that the Kaiser model can not only be applied to the overall emergency management of the hospital, but also can be used to analyze the vulnerability of key departments, key links, and key projects in the hospital. The analysis results play a guiding role in the emergency management of relevant departments of the hospital [12].

Hospital disaster vulnerability analysis is a difficult point in risk assessment. Using this model to conduct scientific disaster vulnerability analysis in hospital emergency management and risk management has become a hot spot, not the end but the starting point for a new round. It is suggested that hospitals can use this model to analyze each risk according to regional characteristics and actual conditions, prioritize planning, strengthen training, avoid disasters and minimize damage after disasters.

\section{Acknowledgement}

Supported by Shaanxi Province Key Research and Development Program (International Cooperation) in 2020 (China - Turkish Medical and Nursing Integrated Multidisciplinary Child Health Research, Project No. 2020KW-0520).

\section{Conflict of Interest}

No conflict of interest.

\section{References}

1. ASHE (2001) American Society for Health-care Engineering of the American hospital Association. Chicago, USA.

2. Cao Jiaqin, Deng Jianhong, Wang Haiyan (2017) Several studies on disaster rescue nursing training recommendations. Journal of Practical Clinical Nursing 2(40): 185-187.

3. Wei Runling, Zhai Shuhui, Yang Yajing (2015) The application of Caesar model in hospital disaster vulnerability analysis. Journal of Traditional Chinese Medicine Management 23(10): 14-16.

4. Gao Rui (2015) Experience in first aid nursing in catastrophic events. Chinese Health Standard Management 6(6): 12-13.

5. Dong Enhong, Liu Huali, Qian Zhiwang (2018) Based on Kaiser model, pediatric nursing risk event identification and evaluation and improvement strategies in a certain city in Shandong. Chinese General Practice 16(7): 1204-1207.

6. Zhang Yan (2019) Analysis of disaster vulnerability of hospital nursing work based on Kaiser model. Transportation Medicine 33(5): 528-531.

7. Dong Jun, Zhou Yachun (2013) Practice of Chinese hospital review. Beijing: People's Military Medical Press.

8. Yin Yixia, Lin $\mathrm{Xu}, \mathrm{Ou}$ Lingbing (2013) Carry out disaster vulnerability analysis and strengthen hospital emergency management. Youjiang Medicine 41(1): 126-128.

9. Ma Xin, Mao Huiping, Zhang Zeyue (2015) The application of PDCA cycle in the improvement of hospital emergency management. Chinese Health Quality Management 22(1): 130-132.

10. Zhang Yan, Gao Yuming, Wang Wei (2016) Analysis of hospital disaster vulnerability. China Health Industry 13(15): 31-33.

11. Chen Jin, Gui Hongbin, Yan Ruoyu (2014) Analysis of hospital disaster vulnerability based on Kaiser model. Chinese Health Quality Management 21(3): 77-79.

12. Liu Siyan, Ji Guozhong, Ma Jianxin (2019) Disaster vulnerability analysis of a foreign hospital in Jiangsu Province based on the KAISER model. Jiangsu Health Service Management 30(12): 1543-1545. 